\title{
Análisis de interferencias léxicas y morfosintácticas en las producciones escritas de alumnos de bachillerato de $\mathrm{E} / \mathrm{LE}$
}

\section{Analysis of lexical and morphosyntactic interferences in the written productions of E/LE baccalaureate students.}

DOI: $10.46932 / \mathrm{sfjdv} 2 \mathrm{n} 2-182$

Received in: March 1st, 2021

Accepted in: May 30th, 2021

\author{
Stéphanie Messakimove \\ Enseignant-chercheur \\ École Normale Supérieure / CRAAL \\ Libreville Gabon \\ E-mail: masthebony@yahoo.fr
}

\section{RESUMEN}

Nuestra experiencia como docente y examinadora de la prueba escrita de español como lengua extranjera (E/LE) en el Baccalauréat ${ }^{l}$ nos ha permitido constatar que las producciones escritas de los alumnos presentan determinadas carencias al nivel lingüístico, lo que dificulta la comprensión de sus textos en esa lengua. El corpus de datos de esta investigación está constituido por los errores relativos a las producciones escritas que realizaron los alumnos en el examen final de baccalauréat de la convocatoria 2013. A través del análisis de errores identificados en 150 textos de alumnos, en el presente trabajo se reflexiona sobre las causas variadas de los errores y sobre la interferencia de la lengua materna, el francés. Para analizar las interferencias lingüísticas que aparecen en sus producciones se tomó como metodología el análisis de errores (Corder, 1967), que nos llevó primero a identificar el error, luego a describirlo y finalmente a intentar explicar su origen. Los resultados indican que algunas interferencias lingüísticas de nuestros alumnos son más cercanas del francés que del español (Eyeang y Messakimove, 2015). Otras reflejan más una lengua intermedia el "FRANPAÑOL", un idioma artificial mezcla de FRANCÉS y ESPAÑOL (Martín Franco, 1990). Los resultados de este estudio pueden servir para remediar el material y las técnicas de enseñanza con el objeto de disminuir esta distancia.

Palabras clave: Análisis de errores, expresión escrita, interferencias lingüísticas, evaluación.

\section{ABSTRACT}

Our experience as a teacher and examiner of the written test of Spanish as a foreign language (E/LE) in the Baccalauréat has allowed us to verify that the written productions of the students present certain deficiencies at the linguistic level, which hinders the comprehension of their texts in that language. The corpus of data of this research is constituted by the errors related to the written productions made by the

\footnotetext{
${ }^{1}$ En el sistema educativo gabonés el Baccalauréat, que se suele llamar el BAC, son los exámenes que sirven para obtener el título de bachillerato al final de la enseñanza secundaria y que son condición indispensable para acceder a la universidad. Son dos grupos de pruebas (10 u 11 asignaturas) organizadas sucesivamente:

- las pruebas del primer grupo (escritas para todas las materias, a excepción de las lenguas donde los candidatos pueden elegir entre las dos modalidades), obligatorias para todos los candidatos al final del tercer curso del liceo;

- las pruebas del segundo grupo (pruebas orales de recuperación), que han de pasar solo los candidatos que hayan obtenido una nota media inferior a 10/20, e igual o superior a 8, en las pruebas del primer grupo. Han de elegir dos asignaturas que son objeto de una prueba escrita en el primer grupo (la calificación se expresa entre 0 y 20). El sistema de calificación consiste en obtener una media de 10/20 o más para ser admitido. El organismo evaluador se compone de un jurado designado por la "Direction Générale des Examens et Concours". En Gabón, el baccalauréat constituye el primer título de la Enseñanza superior.
} 
students in the baccalauréat final exam of the 2013 convocation. Through the analysis of errors identified in 150 students' texts, the present work reflects on the varied causes of errors and on the interference of the mother tongue, French. In order to analyze the linguistic interferences that appear in their productions, the methodology used was error analysis (Corder, 1967), which led us first to identify the error, then to describe it and finally to try to explain its origin. The results indicate that some linguistic interferences of our students are closer to French than to Spanish (Eyeang and Messakimove, 2015). Others reflect more an intermediate language the "FRENCH", an artificial language mixture of FRENCH and SPANISH (Martin Franco, 1990). The results of this study can be used to remediate the material and teaching techniques in order to reduce this gap.

Key words: Error analysis, written expression, linguistic interference, evaluation.

\section{INTRODUCCIÓN}

En el marco de la adquisición de segundas (ASL) el "Error es un fenómeno inevitable en el aprendizaje de lenguas modernas" (Corder, 1967). De manera general, suele haber dos actitudes contrarias ante el concepto de error: una negativa y otra positiva. La actitud negativa, por un lado, ve el error como un elemento que afecta al proceso de aprendizaje y aleja al estudiante del nivel deseado de competencia en L2 (Astolfi, 2003). Por otro, la actitud positiva considera el error como parte del proceso de aprendizaje e indicación de las distintas etapas de aprendizaje que atraviesa el estudiante (Vázquez, 1991; Santos Gargallo, 1993). Son tres los modelos de análisis de errores de los alumnos: el Análisis Contrastivo, el Análisis de Errores y la Interlengua que se describen a continuación.

El modelo del Análisis Contrastivo (AC, 1945-1967) cuyos precursores son Fries (1945) y Lado (1987) se fundamenta en la comparación sistemática y sincrónica de dos sistemas lingüísticos, el sistema de la lengua nativa (L1) y el sistema de la lengua meta (L2) para luego determinar las diferencias y similitudes entre ambos sistemas, y así se podrán predecir las áreas de dificultad en el aprendizaje de una determina L2. Dos conceptos importantes: Interferencia = fenómeno lingüístico por el un rasgo fonético, morfológico, sintáctico o léxico de la lengua materna aparecerá en la estructura de la lengua empleada por el estudiante en el proceso de aprendizaje de una L2. Error = desviación de la norma de la lengua meta, originado por interferencia con la estructura de la lengua nativa. La Metodología consiste en: a) descripción estructural de L1 y L2; b) elaboración de listado de estructuras no equivalentes; c) establecimiento de 1a jerarquía de dificultad; d) predicción y descripción de las dificultades en el aprendizaje; e) elaboración del material didáctico Críticas: a) confusión entre explicación y descripción;

b) falta de precisión en la descripción de los errores; c) simplismo en la subcategorización de los errores, entre varias.

El modelo del Análisis de Errores (AE) de Corder (1967 -1972) se fundamentó en el estudio empírico y sistemático de los errores producidos por estudiantes de una L2. Los conceptos fundamentales 
son Error = desviación sistemática y recurrente que afecta a la norma estándar de la lengua meta, es positivo e inevitable, y evidencia que el proceso de aprendizaje se está produciendo. Dialecto transitorio = sistema lingüístico que emplea el estudiante de una L2, que presenta características de la lengua nativa (L1), de la lengua meta (L2 características idiosincrásicas. ) y otras. a) identificación de los errores; b) clasificación de los errores de acuerdo con la taxonomía previamente determinada; c) descripción de los errores Críticas: a) escasa aplicabilidad en el enseñanza; c) abstracción en las descripciones; d) limitación en la consideración de la causa del error; e) fallo en las predicciones.

El modelo de Interlengua (IL) de Slinker $(1972,1993)$ tiene por objetivos describir el sistema de reglas de un individuo que aprende y se comunica en una LE; y poner de relieve los mecanismos psicolingüísticos que subyacen a la IL y permiten su desarrollo. Los conceptos fuertes son: Interlengua = sistema lingüístico empleado por el estudiante de una L2, que media entre el sistema de la lengua nativa (L1) y el de la lengua meta (L2). Sistema autónomo. Folización = fenómeno lingüístico que caracteriza la interlengua, y que hace mantener rasgos de la gramática de la lengua materna en la estructura del sistema empleado por el estudiante. Estrategias de comunicación = fenómeno lingüístico resultado de planes conscientes que el estudiante elabora para resolver un determinado problema de comunicación ante la carencia lingüística. La Metodología consiste en: a) determinación del perfil del estudiante; b) determinación del tipo de análisis (longitudinal o transversal), d) diseño de la tarea. Críticas: a) escasez de estudios empíricos; c) aparato teórico resbaladizo (Santos Gargallo, 1993).

En este estudio se ha analizado un corpus de redacciones pertenecientes a la prueba escrita de español (PEE) en los exámenes del Baccalauréat, con el objetivo de estudiar y evaluar su competencia lingüística en E/LE a través de los errores léxicos y morfosintácticas más recurrentes. El objetivo general es analizar los errores más frecuentes en un grupo de alumnos para determinar cuáles son las áreas de mayor dificultad en el aprendizaje del español como lengua extranjera; identificar el error, describirlo y explicar su origen. ¿Qué categorías de errores son frecuentes en los textos de los alumnos en la prueba escrita de español en el baccalauréat? ¿Cómo interfiere el francés LM entre el vocabulario y las estructuras?

\section{METODOLOGÍA}

En Gabón, el requisito fundamental para acceder al primer ciclo de enseñanza superior es haber superado las pruebas del Baccalauréat. En estos exámenes, los alumnos están siendo evaluados en todas las materias del currículo del último curso de bachillerato. El corpus de datos lo constituye un conjunto de ciento cincuenta redacciones escritas realizadas por los alumnos de bachillerato en español de la convocatoria 2013. Son alumnos de edades comprendidas entre 16 y 20 años de ambos sexos. Su 
formación académica respondía en unos casos a cuatro y en otros a cinco cursos de español, como asignatura del currículo (tres horas semanales de instrucción reglada). El perfil de los informantes es el siguiente:

- Formación en español llevada a cabo por profesorado no nativo. Nivel de contacto de los alumnos con la lengua objeto de estudio y el grado de exposición a la misma se limitan al aula.

- Son alumnos que aprenden español en un contexto de diglosia favorable al francés: una cincuentena de lenguas locales sin estatus oficial y una lengua oficial de trabajo, el francés (Constitución de 1991). Algunos tienen al francés L1 y L2 una lengua autóctona o de algunos países africanos ${ }^{2}$, otros sólo tienen al francés como L1/LM, pocos tienen al francés L2 y además, las lenguas locales se adquieren en el entorno familiar porque no son objeto de enseñanza. La lengua de primera socialización de nuestros informantes es el francés que es al mismo tiempo lengua de instrucción, lengua vehicular y materia de aprendizaje (Messakimove, 2009).

Para analizar las interferencias lingüísticas que aparecen en las producciones escritas de los alumnos se tomó como metodología el Análisis de Errores (AE) que se desarrolla en tres pasos que a continuación describimos (Corder, 1967, 1981): a) identificación del error, b) descripción del error y, c) explicación del error. Hemos tenido en cuenta, también, la sistematización que Santos Gargallo (2009) hace de los procedimientos del AE. Se proponía el estudio y análisis de los errores cometidos por los aprendices de segundas lenguas para descubrir sus causas y conocer las estrategias que utilizan los alumnos en el proceso de aprendizaje.

a) Identificación del error. El primer paso en la realización de un análisis de los errores es detectar los errores. Uno de los objetivos del AE es establecer un inventario de los errores más frecuentes. Identificar un error nos es tarea fácil, puesto que exige del investigador la reconstrucción de las intenciones comunicativas del alumno y pone de manifiesto la gramática de su propia IL.

b) Descripción del error. Tras identificar el error, no siempre es fácil delimitarlo. Después de detectar el error, el paso siguiente suele ser la categorización de los errores para su descripción posterior. Para hacer una clasificación de los errores, primero es necesario diseñar un instrumento metodológico que comprenda las categorías de los errores que se pretende analizar. En esta fase, hemos clasificado los errores tomando como referencia el criterio etiológica-lingüística propuesto por Santos Gargallo (2009, p. 95) que hace una clasificación de los errores basada en el concepto de transferencia lingüística, lo que nos lleva a dos tipos de errores: (i) Interlingüísticos: aquellos errores causados por interferencia con la lengua nativa del

\footnotetext{
${ }^{2}$ En el contexto sociolingüístico de Gabón las lenguas locales coexisten con algunas lenguas de inmigrantes de África del oeste (Senegal, Togo, Benin).
} 
estudiante o con otras lenguas que ha aprendido anteriormente, (ii) Intralingüísticos: errores debidos al efecto de la lengua meta en sí misma sobre el proceso de aprendizaje.

Del mismo modo que es difícil identificar el error, también es difícil describirlo. Los errores a nivel de texto pueden ser de léxico o de gramática y en el nivel del discurso son los errores de coherencia. En el caso concreto de nuestro trabajo, el objetivo es la descripción de la interlengua francés-español de aprendices de nivel intermedio. Evidentemente, esta descripción no va a ser completa, sino que se centrará solamente en los fenómenos más representativos, por su recurrencia en las producciones de los alumnos. c) Explicación del origen del error. Esta fase destinada a determinar cuál es la causa de un error constituye uno de los aspectos más importantes. Con la fase de explicación de los errores, la metodología del AE intenta establecer la fuente del error. Sin embargo, es la fase que plantea mayor dificultad porque existen varios fuentes de los errores. El objetivo aquí es fundamentar el motivo por el cual se produce un error. El análisis de los errores tanto formales como semánticos siguiendo el criterio etiológico (Vázquez, 1991), criterio etiológico-lingüístico y gramatical (Santos Gargallo, 2009) y criterio de la descripción lingüística (Fernández Jódar, 1996), permite conocer si estos tipos de errores se deben a la transferencia (error interlingüístico) o al propio proceso de desarrollo de la interlengua (error intralingüístico).

Para el análisis, hemos seguido la clasificación según el subsistema lingüístico afectado. Las interferencias más representativas en nuestro corpus de datos son las siguientes: léxicas y morfosintácticas. El término "interferencia" es un concepto que describe/explica de forma acertada varias procesos que tienen lugar en el aula. En didáctica de L2/LE, es la desviación de la norma lingüística del idioma que se está aprendiendo motivada por influencia de estructuras aprendidas con anterioridad.

Tabla 2.1. Categoría y subcategorías de las interferencias analizadas (elaboración propia)

\begin{tabular}{|l|l|}
\hline \multicolumn{2}{|l|}{ CATEGORÍAS Y SUB-CATEGORÍAS DE LAS INTERFERENCIAS ANALIZADAS } \\
\hline Léxicas & Morfosintácticas \\
\hline - Falsos amigos & - Artículos \\
- Calcos & - Preposiciones \\
- Barbarismos & - Verbos \\
\hline
\end{tabular}

Tabla 2.2. Modelo de plantilla de análisis de categorías (Adaptado de Santos Maldonado, 2002)

CATEGORÍA LINGÜÍSTICA: Clasificación general de las interferencias según criterios formales, en los niveles que definen la lengua como sistema.

TIPO DEL ERROR: Denominación de la interferencia según su incidencia predominante en una categoría lingüística determinada.

\begin{tabular}{|c|c|c|}
\hline DESCRIPCIÓN & EJEMPLOS & EXPLICACIÓN \\
\hline $\begin{array}{l}\text { Modo de manifestarse el error } \\
\text { interferencial según su alteración } \\
\text { formal en el enunciado, teniendo en } \\
\text { cuenta la relación contrastiva } \\
\text { francés lengua materna/ español } \\
\text { lengua extranjera (FLM/ELE) y la } \\
\text { intención significativa del alumno. }\end{array}$ & $\begin{array}{l}\text { Ilustran el tipo de interferencia } \\
\text { y su corrección, considerando } \\
\text { los aspectos de campo y de } \\
\text { extensión del error. }\end{array}$ & $\begin{array}{l}\text { Procesos subyacentes al tipo de } \\
\text { interferencia analizado: causas del } \\
\text { error, a partir de la intención } \\
\text { significativa del alumno en el francés } \\
\text { lengua materna (FLM). }\end{array}$ \\
\hline
\end{tabular}




\section{RESULTADOS}

El análisis de las producciones escritas de los alumnos nos ha permitido diagnosticar cuáles son los aspectos que ofrecen un mayor grado de complejidad para los hablantes de francés que aprenden español y señalar algunas incorrecciones gramaticales y léxicas en el uso del español por analogía con construcciones de la lengua francesa. Muchos de estos errores son presentes en los textos de la prueba escrita, las cuales revelan que algunas faltas de ortografía de los alumnos francófonos se producen por el calco con palabras equivalentes de su lengua materna. Veamos, por lo tanto, las dificultades inherentes al empleo del español para estos hablantes de francés, mediante una descripción de los problemas observados en los distintos niveles del análisis lingüístico: léxico y morfosintáctico.

\subsection{ANÁLISIS DE LAS INTERFERENCIAS LÉXICAS}

Muchos estudios han puesto de relieve una interrelación entre tener un vocabulario amplio y la comprensión de una lengua extranjera. El Marco común (2002) define la competencia léxica como “el conocimiento del vocabulario de una lengua y la capacidad para utilizarlo, se compone de elementos léxicos y elementos gramaticales" (p. 108). La falta de vocabulario o el poco dominio de la competencia léxica constituye, desde luego, un verdadero obstáculo para el desarrollo de una verdadera competencia comunicativa.

Como lenguas vecinas (Dabéne, 1975), el francés y el español tienen amplísimas zonas de coincidencia y a la vez una gran cantidad de falsos amigos, que pueden provocar numerosos malentendidos en la comunicación pares archiconocidos como adiós / adieu ('adiós para siempre'), constipado / constipé ('estreñido'), entender / entendre ('oír'), demandar / demander ('preguntar' o 'pedir'), discutir / discuter ('hablar', 'charlar'), embarazo / embarras ('incomodidad', 'molestia'), habitación / habitation ('vivienda') ... son solo unos pocos ejemplos representativos (Santos Maldonado, 2002). Veamos ahora las interferencia léxicas con mayor frecuencia de uso en los textos de nuestros alumnos.

Tabla 3.3. Interferencias léxicas

\begin{tabular}{|c|c|c|}
\hline \multicolumn{3}{|c|}{ INTERFERENCIAS LÉXICAS } \\
\hline DESCRIPCIÓN & EJEMPLOS & EXPLICACIÓN \\
\hline $\begin{array}{l}\text { FALSOS AMIGOS: } \\
\text { palabras que en distintos } \\
\text { idiomas tienen una forma } \\
\text { parecida, debida a un origen } \\
\text { común, pero una significado } \\
\text { diferente, debido a su } \\
\text { desarrollo posterior en } \\
\text { ambientes distintos. }\end{array}$ & $\begin{array}{l}\text {-*progressión del fr. } \\
\text { progression) } \Rightarrow \text { esp. progreso } \\
\text { - *demandar del fr demander } \Rightarrow \\
\text { esp pedir, preguntar } \\
\text { - *progressar del fr. progresser } \\
\Rightarrow \text { esp. avanzar } \\
\text { - *preguntar / entendre (oír) }\end{array}$ & $\begin{array}{l}\text { Los falsos amigos y la presencia de amplias } \\
\text { zonas de coincidencia son responsables de } \\
\text { numerosos malentendidos en la } \\
\text { comunicación }\end{array}$ \\
\hline CALCOS: & - *el auteur $\Rightarrow$ esp.el autor & \\
\hline
\end{tabular}




\begin{tabular}{|c|c|c|}
\hline $\begin{array}{l}\text { traducción palabra por palabra } \\
\text { de una frase o locución } \\
\text { extranjera, o traducción de los } \\
\text { elementos formantes de una } \\
\text { palabra, construida con } \\
\text { elementos existentes en el } \\
\text { idioma ai que traduce. }\end{array}$ & $\begin{array}{l}\text { *en effecto (en effet) } \Rightarrow \text { esp en } \\
\text { efecto } \\
\text { - *differentes } \Rightarrow \text { esp. diferentes } \\
\text { (influencia del francés, } \\
\text { différents) } \\
\text { - * connocimientos } \Rightarrow \text { esp. } \\
\text { conocimientos (a partir del } \\
\text { francés connaissances) } \\
\text { - *apprender } \Rightarrow \text { esp. aprender } \\
\text { (fr. apprendre) } \\
\text { *occasión } \Rightarrow \text { esp. ocasión (fr. } \\
\text { occasion) } \\
* \text { apportar del aportar fr } \\
\text { apporter } \Rightarrow \text { esp aportar }\end{array}$ & $\begin{array}{l}\text { El calco con la grafía habitual de la lengua } \\
\text { francesa explica las faltas de ortografía en } \\
\text { la escritura de muchas palabras del español } \\
\text { con consonantes dobles y en otras palabras } \\
\text { calcadas del francés. } \\
\text { - Conservación de la doble consonante de la } \\
\text { LM en la LE }\end{array}$ \\
\hline $\begin{array}{l}\text { BARBARISMOS: } \\
\text { son incorrecciones que } \\
\text { consisten en pronunciar o } \\
\text { escribir mal las palabras, o en } \\
\text { emplear vocablos impropios } \\
\text { (RAE). }\end{array}$ & $\begin{array}{l}\text { *recherchos en lugar de } \\
\text { investigaciones > fr. recherches } \\
\text { *microbes en lugar de microbios } \\
\text { > fr. microbes } \\
\text { *su disparución en lugar de } \\
\text { desaparición >fr. sa disparition } \\
\text { *chimicos en lugar de químicos } \\
\text { >fr. chimiques } \\
\text { *guerrison en lugar de } \\
\text { recuperación > fr. guérrison } \\
\text { *mecontantementé en lugar de } \\
\text { descontento } \\
\text { mécontentement } \\
\text { *el medio de ralentir en lugar de } \\
\text { ralentizar }\end{array}$ & $\begin{array}{l}\text { Aunque las relaciones léxicas del francés y } \\
\text { del español favorecen la comprensión } \\
\text { global de documentos por parte de los } \\
\text { aprendices francófonos de ELE, hemos } \\
\text { documentado errores de expresión } \\
\text { influenciados por el peso de la lengua } \\
\text { materna de los estudiantes. } \\
\text { Los barbarismos son frecuentes a partir de } \\
\text { palabras francesas, ya sean sustantivos, } \\
\text { verbos o adverbios. }\end{array}$ \\
\hline
\end{tabular}

Como puede notase, las producciones escritas de los alumnos muestran una pobreza de vocabulario que tiene como consecuencia la experimentación de enormes dificultades para un uso comunicativo de la lengua de estudio. Los calcos de la lengua nativa en la L2 es un hecho tan evidente en un escritor ineficiente. A la hora de redactar, el aprendiz de una LE recurre a menudo a la LM que domina mejor. Traduce expresiones o frases enteras de su lengua a la lengua de aprendizaje. La traducción "palabra por palabra" dará probablemente transposiciones erróneas, sobre todo cuando ambas lenguas son vecinas (Dabène, 1975; Sánchez Iglesias, 2003), como el caso del francés y el español.

\subsection{ANÁLISIS DE LAS INTERFERENCIAS MORFOSINTÁCTICAS}

El sistema verbal del español es bastante complejo para quien lo aprende como una segunda lengua (Santos Maldonado, 2002). También en las producciones escritas de los alumnos aparecen ciertas realizaciones que divergen de las del español normativo (RAE, 2014): artículos, preposiciones, verbos.

\section{a) Artículos}

Como se observa en la tabla a continuación, la búsqueda de equivalencias con su lengua materna es fuente de algunas equivocaciones en las producciones de los aprendices francófonos. 
Tabla 3.4. Análisis de las interferencias ortográficas

\begin{tabular}{|c|c|c|}
\hline \multicolumn{3}{|c|}{ ARTÍCULOS } \\
\hline DESCRIPCIÓN & EJEMPLOS & EXPLICACIÓN \\
\hline $\begin{array}{l}\text { Cambio de género: } \\
\text { Cambio de género en } \\
\text { sustantivos franceses de } \\
\text { uso muy frecuente y, como } \\
\text { consecuencia, en los } \\
\text { determinantes y adjetivos } \\
\text { que acompañan }\end{array}$ & $\begin{array}{l}\text { *la planeta (del fr la planète) } \Rightarrow \text { esp el } \\
\text { planeta } \\
\text { *el solución (fr. la solution) } \\
\text { *los actividades (fr. les activités) } \Rightarrow \text { esp.las } \\
\text { actividades } \\
\text { *este enfermedad (fr. cette maladie) } \Rightarrow \text { esp } \\
\text { esta enfermedad } \\
\text { *un palabra (fr. un mot) } \\
\text { *el problemo (fr le problème) } \\
\text { *los capacidades (fr. les capacités) } \Rightarrow \text { esp } \\
\text { las capacidades }\end{array}$ & $\begin{array}{l}\text { En el género de los artículos que } \\
\text { preceden a sustantivos del español, la } \\
\text { búsqueda de equivalencias con su } \\
\text { lengua materna es fuente de algunas } \\
\text { equivocaciones en las producciones } \\
\text { de los aprendices francófonos. }\end{array}$ \\
\hline $\begin{array}{l}\text { Artículo partitivo } \\
\text { Uso del artículo partitivo } \\
\text { cuando se quiere indicar } \\
\text { una parte del sustantivo } \\
\text { de referencia }\end{array}$ & $\begin{array}{l}\text { *participar a al destrucción *del } \\
\text { *populación } \Rightarrow \text { fr de la population } \\
\text { - *sera la causa *de el calentamiento (del } \\
\text { * a al bienestar (al } \\
* \text { del enfermedad (de la } \\
\text { *de el científico en vez de } \\
\text { * del transformación }\end{array}$ & $\begin{array}{l}\text { Condicionamiento morfológico del } \\
\text { FLM donde, generalmente, se } \\
\text { manifiesta la marca del partitivo. }\end{array}$ \\
\hline $\begin{array}{l}\text { Omisión del } \\
\text { artículo }\end{array}$ & $\begin{array}{l}* \text { es la madre de todas ciencias } \Rightarrow \text { esp } \\
\text { todas las ciencias } \\
* \text { detecta todas enfermedades } \Rightarrow \text { esp todas } \\
\text { las enfermedades/toda enfermedades }\end{array}$ & $\begin{array}{l}\text { La influencia del francés se advierte } \\
\text { igualmente en la omisión indebida } \\
\text { del artículo en estructuras locativas } \\
\text { como en los ejemplos mencionados }\end{array}$ \\
\hline
\end{tabular}

\section{b) Preposiciones}

Las preposiciones son muy difíciles de aprender en todas las lenguas, raras veces se encuentra coincidencia entre las de una lengua y otra. Muchas veces en caso de desconocimiento los alumnos de español recurren a la traducción de la preposición que usarían en la lengua materna en un contexto similar. En el nivel morfosintáctico, destacan las confusiones en el régimen preposicional de los verbos por el influjo analógico con las estructuras de la lengua francesa. Entre los errores más frecuentes de los hablantes de francés se encuentran los relacionados con las preposiciones a y en. Otro error corriente entre los aprendices francófonos de ELE es el uso de la preposición por en lugar de para, probablemente motivado por la mayor proximidad fonética de la primera con el francés pour: así, en las correcciones del examen, hemos constatado el empleo indebido de por para aludir al destinatario (*por mí > fr. pour moi) o indicar la finalidad (* somos por luchar $>$ fr. nous sommes pour lutter).

Notamos que el sistema preposicional es donde destacan confusiones sobre todo en el régimen preposicional de los verbos, debidas al influjo analógico con las estructuras con la lengua francesa. Los errores más frecuentes de los alumnos de bachillerato están relacionados con la par preposicional por y para (Santos Maldonado, 2002). 
Tabla 3.5. Interferencias morfosintácticas: Preposiciones

\begin{tabular}{|c|c|c|}
\hline \multicolumn{3}{|c|}{ PREPOSICIONES } \\
\hline DESCRIPCIÓN & EJEMPLOS & EXPLICACIÓN \\
\hline \multicolumn{3}{|l|}{$A$} \\
\hline $\begin{array}{l}\text { Omisión de la } \\
\text { preposición }\end{array}$ & $\begin{array}{l}\text { *informar la populación } \Rightarrow e s p \\
\text { informar a la población }\end{array}$ & $\begin{array}{l}\text { Influencia de la estructura francesa del verbo } \\
\text { informer que es un verbo transitivo, por lo tanto } \\
\text { no necesita un COI como en español. }\end{array}$ \\
\hline $\begin{array}{l}\text { Perífrasis verbales } \\
\text { para expresar la } \\
\text { continuidad }\end{array}$ & $\begin{array}{l}\text { *los científicos continuan *a trabajar } \\
\Rightarrow \text { esp .los científicos continuan } \\
\text { trabajando }\end{array}$ & $\begin{array}{l}\text { Tansferencia de una estructura que no se domina } \\
\text { en la L1 a la L2: } \\
\text { Les scientifiques continuent de travailler. } \\
\Rightarrow \text { esp .los científicos continuan trabajando } \\
\text { (continuar + gerundio) }\end{array}$ \\
\hline \multicolumn{3}{|l|}{ POR, PARA } \\
\hline $\begin{array}{l}\text { Confusión entre por } \\
\text { y para }\end{array}$ & $\begin{array}{l}\text { - *por mí } \Rightarrow \text { fr. Pour moi; } \\
\text { - *la ciencia y la tecnología } \\
\text { constituyen una paradoja por -la } \\
\text { sociedad } \Rightarrow \text { fr. Constituent un } \\
\text { paradoxe pour la société (para aludir al } \\
\text { destinatario); } \\
\text { - *por Carl Sagan, la ignorancia de } \Rightarrow \\
\text { fr. Pour Carl Sagan/selon ( } \\
\text { - *voy por estudiar } \Rightarrow \text { fr. Je vais pour } \\
\text { étudier (para indicar la finalidad). } \\
\text {-*también por evitar } \Rightarrow \text { también para } \\
\text { evitar } \\
\text { *para ejemplo } \Rightarrow \text { esp.por ejemplo }\end{array}$ & $\begin{array}{l}\text { Las similitudes de las preposiciones en francés } \\
\text { podrían llevar a los estudiantes a hacer } \\
\text { suposiciones erróneas. } \\
\text { la diferencia entre por y para causa problemas a } \\
\text { nuestros alumnos } \\
\text { Con respecto a los usos de por y para, las } \\
\text { dificultades que se presentan en el aprendizaje } \\
\text { del español como lengua extranjera se } \\
\text { presentaron también en el corpus. }\end{array}$ \\
\hline
\end{tabular}

Además de las influencias del francés, creemos que otra dificultad mayor reside, en parte, en el hecho de no conocer los usos exactos de las preposiciones. La mayoría de los trabajos sobre las dificultades concretas de uso de preposiciones se han centrado en el par preposicional por/para a causa del número importante de errores que produce. Reconociendo la fosilización importante que puede crear este par preposicional, Saínz González (1998) señala que los ejercicios de huecos no ayudan siempre a evitar la ambigüedad ni a clarificar los usos de cada preposición. En proporción menos importante, aparecen errores relacionados con el par preposicional $\boldsymbol{a}$ y $\boldsymbol{e n}$. Los alumnos no son conscientes de que en español los usos de las preposiciones en y a no siempre equivalen a los usos de las preposiciones francesas en y $a$. Se observa muchos: usos de la preposición española $a$ que no coinciden con la preposición francesa $\grave{a}$; usos de la preposición española en que no coinciden con la preposición francesa en.

c) Verbos

En la morfología verbal, el caballo de batalla de los alumnos francófonos es la diferenciación entre ser y estar, puesto que en francés se utiliza être para traducir los dos verbos del español. Entre los errores más generalizados, encontramos el uso incorrecto de ser en lugar de estar en algunas construcciones que vienen consignadas en la tabla a continuación. 
Tabla 3.6. Interferencias morfosintácticas: Verbos

\begin{tabular}{|c|c|c|}
\hline \multicolumn{3}{|c|}{ VERBOS } \\
\hline DESCRIPCIÓN & EJEMPLOS & EXPLICACIÓN \\
\hline $\begin{array}{l}\text { Confusión en el uso de } \\
\text { ser y estar }\end{array}$ & $\begin{array}{l}\text { - *soy de acuerdo/ no es de acuerdo } \Rightarrow \\
\text { fr. Je suis d'accord/ il n'est pas } \\
\text { d'accord. } \\
\text { - nosotros *somos de acuerdo } \Rightarrow \text { fr. } \\
\text { Nous sommes d'accord } \\
\text { - *somos contentos } \Rightarrow \text { fr. nous sommes } \\
\text { contents. } \\
\text { - *es (muy) bien } \Rightarrow \text { fr. C'est très bien } \\
\text { - la ciencia debe *estar una solución } \\
\Rightarrow \text { fr. La science doit être une solution }\end{array}$ & $\begin{array}{l}\text { En el sistema verbal español, la diferencia } \\
\text { entre ser y estar es el rompe cabeza para los } \\
\text { aprendices francófonos, ya que en francés se } \\
\text { utiliza être para traducir los verbos del } \\
\text { español ser y estar. }\end{array}$ \\
\hline $\begin{array}{l}\text { Confusión en el uso de } \\
\text { haber y tener }\end{array}$ & $\begin{array}{l}\text { *la sociedad no ha la seguridad } \\
\text { *los científicos no han la conciencia } \\
\text { la ciencia puede haber } \Rightarrow \text { esp puede } \\
\text { tener (fr la science peut avoir) } \\
\text { *la ciencia ha de los capacidades } \\
\text { *haber una buena hygiene de vida } \\
\text { *la ciencia debe haber el tiempo }\end{array}$ & $\begin{array}{l}\text { Empleo erróneo del verbo haber por tener, } \\
\text { es fruto de la ambivalencia del verbo avoir } \\
\text { en francés. }\end{array}$ \\
\hline $\begin{array}{l}\text { Verbos con preposición } \\
\text { Las preposiciones que se } \\
\text { usan para accompañar a } \\
\text { los verbos en español son } \\
\text { distintas, en muchos } \\
\text { casos, a las que se usan en } \\
\text { francés. }\end{array}$ & $\begin{array}{l}\text { *permite de luchar (fr. permettre de) } \\
\text { *aprovecha de (fr. profiter de) } \\
\text { *los científicos intentan de (fr. essayer } \\
\text { de) } \\
\text { * concibe de }\end{array}$ & $\begin{array}{l}\text { - La preposición } \boldsymbol{d e} \text { ofrece innumerables } \\
\text { ejemplos de errores entre los estudiantes } \\
\text { francófonos de ELE por el calco con } \\
\text { estructuras verbales de régimen } \\
\text { preposicional obligado en la lengua francesa. } \\
\text { Además, la preposición de es más utilizada } \\
\text { en francés que en español, por ejemplo no se } \\
\text { dice "ser adicto de algo" ni "atrasar de diez } \\
\text { minutos", mientras que en francés sí es } \\
\text { posible. }\end{array}$ \\
\hline
\end{tabular}

En las listas de errores característicos de los hablantes de francés, es habitual mencionar la dificultad de distinguir entre los usos de ser y estar. Para los hablantes nativos de francés las principales dificultades se presentan a la hora de diferenciar los verbos ser y estar, al existir en francés el verbo être para expresar el significado de ambos. Además, el verbo avoir es el auxiliar para indicar tanto haber como tener en español. El problema con el que se encuentran es que tienen que asimilar una diferencia que en su L1 no existe; lo que esto da como resultado no es una transferencia directa de las formas y usos del francés, sino un conocimiento vacilante, fundado en una comprensión fragmentaria y simplista. Otro de los grandes problemas que presentan nuestros alumnos con respecto al uso de verbos, es la confusión entre los verbos haber y tener, sobre todo, en el uso de la forma impersonal hay. Ello se debe a que el francés utiliza una forma del verbo tener avoir (il y a) para esta función impersonal.

\subsection{OTRAS INTERFERENCIAS MORFOSINTÁCTICAS RECURRENTES}

El español y el francés, lenguas vecinas por su origen latino, presentan rasgos diferenciadores en la propia estructura morfosintáctica. El francés, al contrario que el español, observa una mayor rigidez en el orden de la frase, rasgo significativo en el aprendizaje. 
Tabla 3.7. Otras interferencias recurrentes

\begin{tabular}{|c|c|c|}
\hline \multicolumn{3}{|c|}{ OTRAS INTERFERENCIAS MORFOSINTÁCTICAS } \\
\hline DESCRIPCIÓN & EJEMPLOS & $\begin{array}{l}\text { EXPLICACIÓN } \\
\end{array}$ \\
\hline $\begin{array}{l}\text { Confusión de } \\
\text { significado }\end{array}$ & $\begin{array}{l}\text { *crear en, en lugar de creer en } \\
\text { *por qué (fr. pourquoi?) en lugar de } \\
\text { porque (fr. parce que) } \\
\text { *para qué (fr.pour quelle finalité?) y } \\
\text { para que (fr. pour telle finalité) } \\
\text { *por que en lugar de porque (fr. parce } \\
\text { que) } \\
\text { Muestras: } \\
\text { - la ciencia y la tecnología constituyen } \\
\text { una paradoja para la sociedad *para } \\
\text { que la ciencia no sólo ha beneficiado a } \\
\text { la humanidad sino que también ha } \\
\text { concebido armas. } \\
\text { - Estoy de acuerdo en primer lugar } \\
\text { *por que los científicos por la } \\
\text { utilización muchos mejores por } \\
\text { estudiar... }\end{array}$ & $\begin{array}{l}\text { Confusión debida a la falta de dominio de uso } \\
\text { de los } 2 \text { verbos. } \\
\text { Confusiones ocasionadas por las carencias de } \\
\text { conocimientos sólidos sobre algunos } \\
\text { elementos del sistema de la lengua. } \\
\text { Por qué: secuencia formada por la preposición } \\
\text { por y el interrogativo o exclamativo qué. La } \\
\text { secuencia por qué no puede sustituirse por } \\
\text { términos como razón, causa o motivo. } \\
\text { Porque: conjunción átona, razón por la que se } \\
\text { escribe sin tilde. Puede usarse con dos valores: } \\
\text { - como conjunción causal, para introducir } \\
\text { oraciones subordinadas que expresan causa; } \\
\text { - como encabezamiento de las respuestas a las } \\
\text { preguntas introducidas por la secuencia por } \\
\text { qués. } \\
\text { Para que: locución conjuntiva para indicar la } \\
\text { finalidad o el propósito de algo. } \\
\text { Para qué: locución. adverbial para qué fin u } \\
\text { objeto. }\end{array}$ \\
\hline $\begin{array}{l}\text { El género y } \\
\text { número de los } \\
\text { nombres }\end{array}$ & $\begin{array}{l}\text { *este enfermedad } \\
\text { *el victoria de la ciencia } \\
\text { *todo los días } \\
\text { *son muy importante }\end{array}$ & $\begin{array}{l}\text { En español, el género y el número de nombres } \\
\text { y adjetivos se forma de un modo distinto que } \\
\text { en francés. Hay nombres con distinto género } \\
\text { en francés y en español. } \\
\text { No asimilación de las reglas de concordancia. }\end{array}$ \\
\hline
\end{tabular}

\section{DISCUSIÓN}

El enfoque más tradicional conocido como modelo orientado al producto explica la naturaleza de la expresión escrita analizando exclusivamente el producto de dicha actividad desde una perspectiva puramente lingüística. A partir de las producciones de los alumnos, hemos identificado los errores lingüísticos: léxicos y morfosintácticas. La recurrencia de ciertos errores nos informa sobre las insuficiencias de los alumnos en la lengua objeto de aprendizaje, en el caso presente, la lengua española. El análisis del corpus de datos muestra que la recurrencia de errores se concentra en categorías gramaticales, en la falta de interiorización de las reglas gramaticales y en interferencia L1 y L2 (francés/español). De manera general, las dificultades que encuentran los alumnos de bachillerato a la hora de producir textos en español se resumen a continuación:

a) Una pobreza en la expresión escrita de las ideas de los alumnos, debida

fundamentalmente a muchas carencias de conocimientos sólidos de los elementos del sistema de la lengua (gramática, léxico, ortográfico). Esta pobreza se presenta, primero, al momento de redactar

\footnotetext{
${ }^{3}$ RAE (2014)
} 
textos y, se manifiesta por la repetición de términos como: *Me acuerdo con, *soy de acuerdo, *me acuerdo, *No hay de acuerdo, *piensamos que, *estoy pienso que, *soy de acuerdo.

b) Y el uso extensivo de algunas palabras que por su amplio espectro de significado se usa como comodines que atentan contra la concisión del enunciado: *phenomeno (fr. phénomène $\Rightarrow$ esp. fenómeno), *telephono portable (fr. téléphone portable $\Rightarrow$ esp. teléfono móvil), *remarquamos (fr. remarquer $\Rightarrow$ esp. notamos), *cherchar (fr. chercher $\Rightarrow$ esp. buscar), *lutar (fr. luter $\Rightarrow$ esp. luchar).

c) También aparecen muchas referencias a la lengua de primera socialización (transferencias lingüísticas), el francés. Algunas producciones de los alumnos son más cercanas del francés que del español (Eyeang y Messakimove, 2015: 419). Es más una lengua intermedia el "FRANPAÑOL” (Martín Franco, 1990), un idioma artificial que es una mezcla de francés y de español, debido a las interferencias lingüísticas como puede observarse en los escritos siguientes: *la ciencia es una factora del destrucción, *a causa del transformación del industrie, *También por evitar mucho los maladías como el sida, los microbes y evitar tambien por una bonna sante.

d) Y en cierta medida la influencia de otra lengua aprendida anteriormente (inglés como primera $\left.\mathrm{LE}^{4}\right): *$ dangerious, *My, *See.

\section{CONCLUSIONES}

En definitiva, se nota una patente deficiencia de los alumnos de bachillerato en la competencia lingüística en español/LE. El discurso de nuestros alumnos aprendices de E/LE se ve afectado por la recurrencia sistemática de errores en determinadas áreas de la gramática que demuestra que las reglas no han sido interiorizadas debidamente. Algunos de esos errores, transferencia negativa y barbarismos (préstamos lingüísticos), rompen la comunicación, obstaculizan la comprensibilidad del mensaje.

Con este estudio hemos conseguido, fundamentalmente dos cosas: saber cómo hablan nuestros alumnos, saber cómo y qué les enseñamos (Santos Gargallo, 2009; Ríos Nava y Olivo Estrada, 2021). ). Tras el análisis, se ha notado cierta distancia entre la interlengua de los alumnos y la lengua meta objeto de estudio. En otras palabras, se deriva una distancia importante entre lo que nosotros profesores enseñamos a los alumnos y sus necesidades reales de comunicación. Algunas interferencias lingüísticas de nuestros alumnos son más cercanas del francés que del español (Eyeang y Messakimove, 2015). Otras reflejan más una lengua intermedia el "FRANPAÑOL", un idioma artificial mezcla de FRANCÉS y ESPAÑOL (Martín Franco, 1990). Los resultados de este estudio pueden servir para remediar el material y las técnicas de enseñanza con el objeto de disminuir esta distancia.

\footnotetext{
${ }^{4}$ En el sistema educativo gabonés, el inglés es la primera lengua extranjera obligatoria en la secundaria.
} 


\section{REFERENCIAS}

Astolfi, J. P. (2003). El "error", un medio para enseñar. (3ª ed.). Diada Editora.

Blanco Picado, A. I. (2002). "El error en el proceso de aprendizaje". Cuadernos Cervantes de la Lengua Española, 38(12-20).

Consejo de Europa. (2002). Marco común europeo de referencia para las lenguas: aprendizaje, enseñanza, evaluación. Anaya e Instituto Cervantes. http://cvc.cervantes.es/ensenanza/biblioteca_ele/marco

Corder, S. P. (1967). The significance of Learners Errors, IRAL 5, pp. 161-170

Corder, S. P. (1981) Error Analysis and Interlanguage. Oxford, Inglaterra: Oxford University Press.

Dabène, L. (1975). L'enseignement de l'espagnol aux francophones (Pour une didactique des langues "voisines"). En E. Koskas y J.-R Ladmiral (Eds.), Langages, (vol. 39, pp. 51-64). Didier-Larousse.

Eyeang, E y Messakimove, S. (2015). "Enfoques didácticos para mejorar la expresión escrita del estudiante gabonés de español lengua extranjera”. In Núnes Sabarís, Xaquín y al (eds), Horizontes científicos y planificación académica en la didáctica de lenguas y literaturas, 409-426. Universidade do Minho: Edicões Hümus.

Fernández Jódar, R. (2006). Análisis de errores léxicos, morfosintácticos y gráficos en la lengua escrita de los aprendices polacos de español (Tesis doctoral). Universidad Adam Mickiewicz, Poznan, Polonia.

Martín Franco, C. (1990). Interferencias lingüísticas en el aprendizaje de español por estudiantes francófonos. Encuentro, Revista de investigación e innovación en la clase de idiomas (3), 117-124. Servicio de Publicaciones de la Universidad de Alcalá. https://ebuah.uah.es/dspace/handle/10017/588?show=full

Messakimove, S. (2009b). Las creencias de los alumnos de la secundaria gabonesa acerca de su proceso de aprendizaje del español/LE, marcoELE (9). https://marcoele.com/las-creencias-de-los-alumnosgaboneses-de-ele/

Real Academia Española (2014). Diccionario de la lengua española. https://www.rae.es/obrasacademicas/diccionarios/diccionario-de-la-lengua-espanola

Ríos Nava, B y Olivo Estrada, J. R. (2021). El estudiante de medicina: sus ideas y renglones. South Florida Journal of Development, Miami, v.2, n.2, $\quad$ p. $1257-1265$. https://southfloridapublishing.com/ojs/index.php/jdev/article/view/380/357

Saínz González, M.E. (1999). La oposición por/para en Español: una nueva propuesta didáctica, Actas del IX Congreso Internacional de ASELE. Español como lengua extranjera: enfoque comunicativo y gramática, Santiago de Compostela, Universidad de Santiago de Compostela, pp. 903-922. https://dialnet.unirioja.es/servlet/articulo?codigo $=892793$

Santos Gargallo, I. (1992). La enseñanza de segundas lenguas. Análisis de errores en la expresión escrita de estudiantes de español cuya lengua nativa es el serbocroata (Tesis doctoral). Universidad Complutense, Madrid, España. 
Santos Gargallo, I. (2009). Análisis Contrastivo, Análisis de Errores e Interlengua en el marco de la Lingüística Contrastiva. Madrid, España: Editorial Síntesis, Colección Lingüística.

Santos Maldonado, Ma. J. (2002). El error en las producciones escritas de francés lengua extranjera: análisis de interferencias léxicas y propuesta para su tratamiento didáctico. (Tesis doctoral). Universidad de Valladolid, Palencia, España.

Sánchez Iglesias, J. (2003). Errores, corrección y fosilización en la didáctica de lenguas afines: Análisis de errores en la expresión escrita de estudiantes italianos de ELE (Tesis doctoral). Universidad de Salamanca, Salamanca, España.

Vázquez, G. E. (1991). Análisis de errores y aprendizaje de español lengua extranjera. Peter Lang. 